\title{
Improving Broadband Displacement Detection with Quantum Correlations
}

\author{
N. S. Kampel,,${ }^{1, *}$ R. W. Peterson, ${ }^{1}$ R. Fischer, ${ }^{1}$ P.-L. Yu, ${ }^{1, \dagger}$ K. Cicak, ${ }^{2}$ R. W. Simmonds, ${ }^{2}$ K. W. Lehnert, ${ }^{1}$ and C. A. Regal ${ }^{1, \star}$ \\ ${ }^{1}$ JILA, University of Colorado and National Institute of Standards and Technology, \\ and Department of Physics, University of Colorado, Boulder, Colorado 80309, USA \\ ${ }^{2}$ National Institute of Standards and Technology, Boulder, Colorado 80305, USA
}

(Received 3 October 2016; published 18 April 2017)

\begin{abstract}
Interferometers enable ultrasensitive measurement in a wide array of applications from gravitational wave searches to force microscopes. The role of quantum mechanics in the metrological limits of interferometers has a rich history, and a large number of techniques to surpass conventional limits have been proposed. In a typical measurement configuration, the trade-off between the probe's shot noise (imprecision) and its quantum backaction results in what is known as the standard quantum limit (SQL). In this work, we investigate how quantum correlations accessed by modifying the readout of the interferometer can access physics beyond the SQL and improve displacement sensitivity. Specifically, we use an optical cavity to probe the motion of a silicon nitride membrane off mechanical resonance, as one would do in a broadband displacement or force measurement, and observe sensitivity better than the SQL dictates for our quantum efficiency. Our measurement illustrates the core idea behind a technique known as variational readout, in which the optical readout quadrature is changed as a function of frequency to improve broadband displacement detection. And, more generally, our result is a salient example of how correlations can aid sensing in the presence of backaction.
\end{abstract}

DOI: 10.1103/PhysRevX.7.021008

When one seeks knowledge of the full dynamics of the displacement of a harmonic oscillator, noncommutation of the two mechanical quadratures requires a minimum added noise equal to the mechanical resonator's zero point motion $[1,2]$. This fundamental quantum limit (QL) is a distinct bound from the standard quantum limit (SQL) that is often considered in interferometric displacement measurement [3-5]. The SQL is a consequence of the noncommutation of the probe's quadratures in a specific measurement configuration and is characterized by a trade-off between shot noise $(\mathrm{SN})$ imprecision and quantum backaction that are uncorrelated [6,7]. The QL and SQL reach the same limit when probing at the peak mechanical response, but the QL can be a significantly lower bound off resonance [Figs. 1(a) and 1(b)]. In studies of micromechanical motion, there has been great interest in observing quantum backaction and approaching the SQL on mechanical resonance [8-12]. However, much of what historically motivates SQL research is displacement monitoring over a wide frequency

\footnotetext{
*nir.kampel@jila.colorado.edu

${ }^{\dagger}$ Current address: School of Electrical and Computer Engineering, Birck Nanotechnology Center, Purdue University, West Lafayette, IN 47907, USA.

${ }^{\ddagger}$ regal@jila.colorado.edu

Published by the American Physical Society under the terms of the Creative Commons Attribution 4.0 International license. Further distribution of this work must maintain attribution to the author(s) and the published article's title, journal citation, and DOI.
}

Subject Areas: Quantum Physics

band, such as gravitational wave searches [13]. One longstanding concept for surpassing the SQL is to introduce correlations by changing the readout quadrature as a function of frequency in a technique known as variational readout $[14,15]$. In the work presented in this article, we measure the displacement of a membrane resonator in an optical interferometer with a tunable readout quadrature. By thermalizing the mechanical device to a dilution refrigerator and mitigating other technical noise sources, we are able to measure quantum noise far off resonance compared to typical micromechanical measurements and achieve near-SQL-limited measurement of a solid-state object. With this starting point we are able to use variational-readout techniques to improve upon the offresonance SQL for our quantum efficiency.

Over the years a variety of techniques have been considered for surpassing the SQL [6], and it is useful to place these in context in comparison to variational readout. Perhaps the most well-known technique is to restrict knowledge to a dynamically decoupled single mechanical quadrature in a quantum nondemolition (QND) measurement to evade backaction completely [16]. Therefore, the total broadband noise can be arbitrarily decreased (up to the zero-point motion) by increasing the probe power. However, QND unfortunately measures only a single phase force, unless the system dimensionality is increased to perform QND measurements on both quadratures [17-20]. While QND measurements have been demonstrated electromechanically [21-24], instabilities can arise, and a measurement below the SQL has not been 
(a)

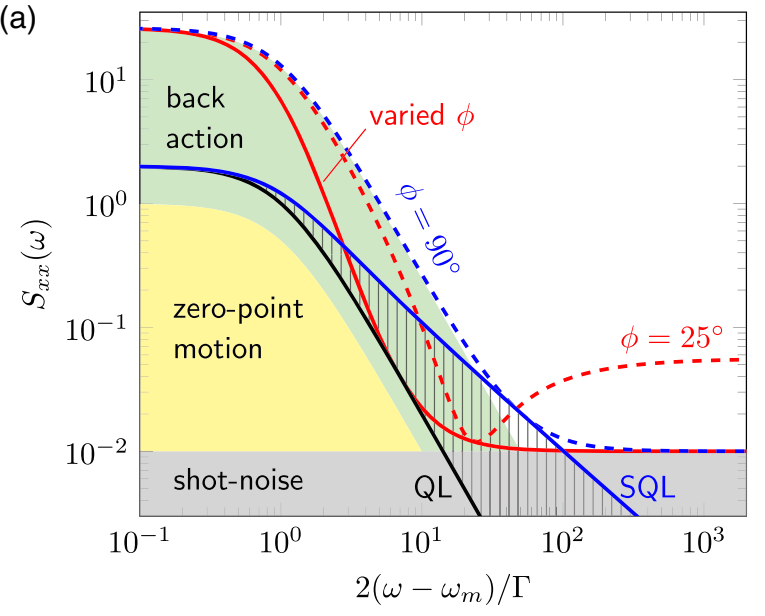

(b)

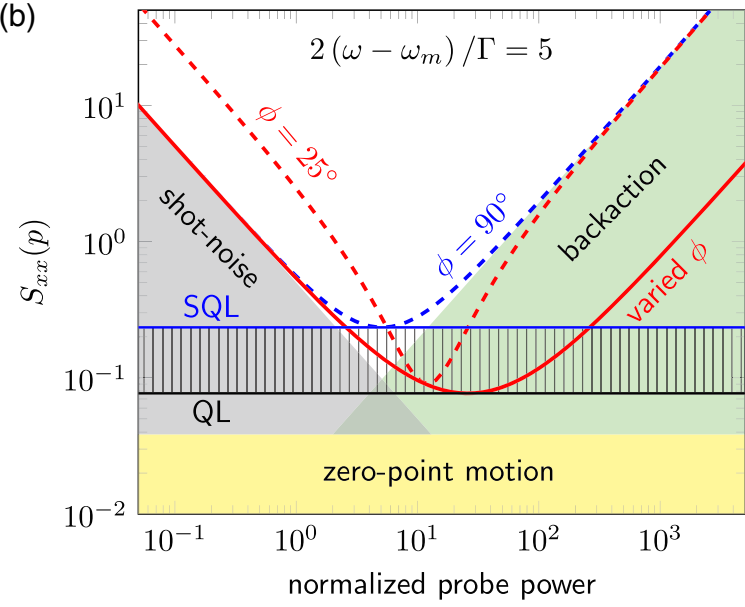

(c)



(d)

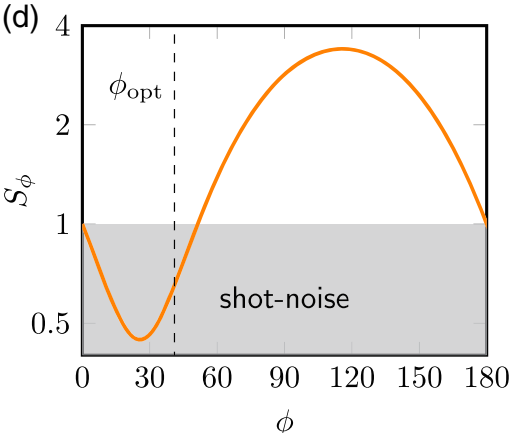

FIG. 1. Imprecision for different interferometric measurement configurations. (a) Frequency dependence for fixed power $p=50$ and (b) power dependence for frequency $2\left(\omega-\omega_{m}\right) / \Gamma=5$. Shaded areas represent the SN (gray), backaction (green), and zero-point motion (yellow). Lines represent measurement at $\phi=90^{\circ}$ (dashed blue), $\phi=25^{\circ}$ (dashed red), variational readout (solid red). The black (QL) and blue (SQL) lines in both (a) and (b) show total limits when the probe power is allowed to vary to optimize total noise, with hatched area highlighting the QL and SQL distinction. All curves are evaluated for ideal quantum efficiency $\epsilon=1$ and zero thermal disturbance. (c) Experimental schematic. Coherent probe beam enters the cavity and interacts with a membrane resonator. The probe light that leaves the cavity is detected via balanced homodyne detection with the measurement angle (quadrature) set by the local oscillator phase $\phi$. (d) Comparison to ponderomotive squeezing for frequency $2\left(\omega-\omega_{m}\right) / \Gamma=5$ and $p=6$. Plotted is the spectral density for the optical output $S_{\phi}$ (orange line) compared to the shot-noise limit (shaded gray) (see Appendix). The dashed black line demonstrates that the optimal mechanical displacement measuring angle $\left(\phi_{\mathrm{opt}}\right)$ is shifted towards the phase quadrature compared to the phase for maximal ponderomotive squeezing.

demonstrated. In our measurements, we focus on a solution in which a two-mechanical quadrature measurement is made, yet quantum correlations between imprecision and backaction are used to address the SQL. Such correlations can be achieved by injecting quadrature squeezed light [15] or using nonlinear cavities $[25,26]$, but an equally capable technique is to rotate the readout quadrature such that the mechanical motion itself mixes the probe's amplitude and phase quadratures [15,27].

For any given frequency, there exists a measurement configuration that can reach the QL for an ideal detector [black line in Fig. 1(a)]. Here, we present an analysis based on the probe's uncertainty relations that reveals this configuration $[3,4,28]$. (Note that the on-resonance SQL can be conveniently arrived at with a Heisenberg microscope argument that dictates a minimum contribution of the shot noise $(\mathrm{SN})$ and backaction [29], but this argument does not hold in the presence of correlation.) We consider the result of a linear measurement of a mechanical harmonic oscillator via an optical probe at a given quadrature angle $\phi$. The analysis is based on an optomechanical interaction in which the probe's phase quadrature linearly depends on the mechanical state, while the amplitude is unchanged. In the analysis of this interaction, we assume a large-photon limit in which the fluctuations are linearized around a large optical field. From the optical probe one can infer a mechanical displacement, and we present our data and theoretical comparison in terms of a dimensionless mechanical displacement. This transformation for an optomechanical interaction in the presence of finite efficiency and conversion to dimensionless units is nontrivial, and the expressions put forth here are derived sequentially in the Appendix to enable concise analysis of their consequences on measurements. 
The dimensionless mechanical displacement inferred from the optical probe can be written as $\hat{X}_{\phi}(\omega)=\hat{x}_{m}(\omega)+$ $\hat{I}_{\phi}-i \tilde{\chi}_{m}(\omega) \hat{F}_{\mathrm{ba}}$. Here, the mechanical state $\left(\hat{x}_{m}\right)$ appears along with $\mathrm{SN}\left(\hat{I}_{\phi}\right)$ and backaction $\left(\hat{F}_{\mathrm{ba}}\right)$, where the $\mathrm{SN}$ and backaction both arise from the probe's fluctuations. The SN is a white noise with a contribution that depends on $\phi$. The backaction term is a result of probe amplitude fluctuations driving the mechanical state and, hence, is a function only of the amplitude quadrature $(\mathrm{AM})\left(\phi=0^{\circ}\right)$. The backaction is filtered by $\tilde{\chi}_{m}(\omega)$, the dimensionless mechanical susceptibility given by $\tilde{\chi}_{m}(\omega)=\left[1-2 i\left(\omega-\omega_{m}\right) / \Gamma\right]^{-1}$, where $\Gamma$ is the effective mechanical linewidth. Within $\hat{x}_{m}$ we include both the zero-point motion and environmental perturbation (thermal and other applied forces).

In the experiment, we measure the symmetrized displacement power spectral density (PSD) $S_{x x}(\omega)=$ $\left\langle\hat{X}_{\phi}(-\omega) \hat{X}_{\phi}(\omega)\right\rangle$ [4], which has contributions from the mechanical resonator $\left(S_{m}\right)$, SN imprecision $\left(S_{I I}\right)$, backaction $\left(S_{F F}\right)$, and their cross-correlation $\left(S_{I F}\right)$

$$
\begin{aligned}
S_{x x}(\omega, p, \phi)= & S_{m}(\omega)+S_{I I}(p, \phi)+\left|\tilde{\chi}_{m}(\omega)\right|^{2} S_{F F}(p) \\
& +2 \operatorname{Im}\left[\tilde{\chi}_{m}(\omega) S_{I F}(\phi)\right] .
\end{aligned}
$$

Throughout, we use dimensionless displacement units such that the zero-point motion contribution to the PSD $S_{x x}$ is 1 on mechanical resonance $\left(\omega=\omega_{m}\right)$, and the probe power $(p)$ is normalized to the SQL power on mechanical resonance. Similarly, the value of the added noise at the SQL (SN and on-resonance backaction) is equal to 1 . In absolute units the added noise at the SQL and the zeropoint motion each contribute $S_{\mathrm{SQL}}\left(\omega_{m}\right)=2 x_{\mathrm{zp}}^{2} / \Gamma$. The zero-point motion is given by $x_{\mathrm{zp}}=\sqrt{\hbar /\left(2 m \omega_{m}\right)}$, where $\omega_{m}$ is the resonant frequency of the mechanical mode of interest, $\hbar$ is the reduced Planck constant, and $m$ is the effective mass of the resonator [9].

From an analysis of the full expression of $S_{x x}$ shown in the Appendix, one finds that the probe's uncertainty relation [28] is connected to the measured PSD via $S_{I I} S_{F F} \geq \frac{1}{4}+S_{I F}^{2}$. The SQL corresponds to the special case in which $S_{I F}=0$, and we find the QL by careful choice of $S_{I F}$ and measurement power for a given frequency $[4,30]$. To understand measurement limits, we plot the contributions to $S_{x x}$ in Fig. 1. First, we describe the lines relevant to SQL physics. The dashed blue line in Fig. 1 shows a phase quadrature (PM) $\left(\phi=90^{\circ}\right)$ measurement for a fixed backaction-dominated probe power, whereas the blue solid line (SQL) results when optimizing the probe power at each frequency. Due to the backaction frequency dependence, the SQL value changes off resonance as $S_{\mathrm{SQL}}(\omega)=S_{\mathrm{SQL}}\left(\omega_{m}\right)\left|\tilde{\chi}_{m}(\omega)\right|$; namely, the SQL value drops and the power required to reach it increases. Figure 1(b) shows that the SQL results in a linear tradeoff between SN and backaction [7].
However, if the homodyne detector is arranged to measure a finite quadrature angle $0^{\circ}<\phi<90^{\circ}$, the cross-correlation term $S_{I F}(\phi)$ in Eq. (1) becomes nonzero and $S_{x x}$ can be smaller than the SQL [Figs. 1(a) and 1(b), dashed red line] $[4,15]$. Because $S_{I F}$ is real, an additional requirement is that $\tilde{\chi}_{m}(\omega)$ has an imaginary part, which happens only off resonance. In particular, $\operatorname{Im}\left[\tilde{\chi}_{m}(\omega) S_{I F}(\phi)\right]$ gives rise to a Fano-like frequency dependence, in analogy to that observed in ponderomotive squeezing of light. While such squeezed light has been observed [31-33], improved measurements were far from accessible in previous experiments due to technical noise at large $\phi$. Figure 1(d) shows for comparison ponderomotive squeezing of the cavity output light. The optimal measurement phase $\left(\phi_{\text {opt }}\right)$ is rotated towards PM compared to the optimal squeezing phase. While rotating towards AM introduces the correlations of interest, it also dilutes mechanical information found only in PM (see the Appendix). In variational readout, $\phi$ is tuned as a function of frequency to approach the QL over a broader range of frequencies [15]. Variational readout at a fixed power is illustrated by the red line of Fig. 1(a). As seen in Fig. 1(b), the power can be optimized in order to reach the QL (solid black line), which corresponds to total noise at twice the zero-point motion.

Variational readout in a homodyne measurement is a technique most suited to broadband, off-resonance displacement or force measurement for $2\left|\omega-\omega_{m}\right| / \Gamma \geq 1$. However, even for on-resonance force measurements quantum correlations can be utilized by employing a two-tone local oscillator, using recently proposed synodyne readout [34]. Synodyne realizes single-quadrature measurement at a given frequency within a range of near-resonant frequencies $\left(2\left|\omega-\omega_{m}\right| / \Gamma \leq 1\right)$. (See the Supplemental Material for an analysis of synodyne and its comparison to the SQL in a frequency-domain picture analogous to Fig. 1 [35].) Variational readout and synodyne are related in that they both approach the readout problem by modifying the local oscillator, instead of, for example, the intracavity field. Lastly, note asymmetric line shapes associated with crosscorrelations have been recently observed in Ref. [36] using an input quadrature squeezed probe in the microwave domain, but an improvement in the off-resonance sensitivity has not been shown to date. Squeezed light can also be used independent of $S_{I F}$ to modify $\hat{I}_{\phi}$ or $\hat{F}_{\mathrm{ba}}$ to modify power requirements $[15,37]$. Injecting squeezed vacuum into the dark port of an interferometer is a technique that has already been implemented in large-scale interferometers (such as advanced LIGO), but their aim was to enable better sensitivity in a fully shot-noise-limited band, without increasing optical power [38].

In our experiments, we create an optical interferometer consisting of a cryogenically compatible Fabry-Perot cavity coupled to a high-stress $\mathrm{Si}_{3} \mathrm{~N}_{4}$ membrane resonator $[33,39,40]$ [Fig. 1(c)]. We probe the motion of the $(2,2)$ membrane mode at $\omega_{m} / 2 \pi=1.596 \mathrm{MHz}$ (twice the 
fundamental frequency), with an exceptionally high-quality factor of $Q_{m}=32 \times 10^{6}$. We optically damp and cool to an effective linewidth of $\Gamma / 2 \pi=340 \mathrm{~Hz}$ by injecting a tone red detuned of the optical cavity. We linearly monitor the displacement for the science described in this work by injecting an on-resonant probe into the cavity. To yield a mechanical spectrum with minimal thermal noise, even a number of mechanical linewidths off resonance, we precool the cavity and membrane to $120 \mathrm{mK}$ and shield the membrane mode by embedding it in a phononic crystal $[41,42]$. At our chosen damping level, we measure via sideband asymmetry a phonon occupation of $n_{\text {th }}=1.29 \pm 0.05$; this final phonon occupation is defined by the optical damping rate we choose, and is not limited by added noise [40] (see Supplemental Material [35]). The outgoing probe light is measured using balanced homodyne detection, with a total quantum efficiency of $\epsilon=0.350 \pm 0.015$. The single-photon coupling is independently calibrated and found to be consistent with a value of $g / 2 \pi=39 \mathrm{~Hz}$ tightly bounded by the experimental data.

We start by measuring $S_{x x}$ on or off resonance at $\phi=90^{\circ}$, in which the measurement noise is similar to the SQL [Fig. 2(a)]. For all frequencies the relative fraction of measurement noise to the SQL at that frequency is constant and equal to 1.7 , as set by our quantum efficiency. For the on-resonance measurements (triangles), a total measured PSD of $5.3 \pm 0.2$ times the on-resonance SQL added noise $\left(S_{\mathrm{SQL}}\right)$ is realized, corresponding to the smallest reported value to our knowledge $[8,11,21]$.
This is due to our low phonon occupation and high quantum efficiency. When we examine the data at $2\left(\omega-\omega_{m}\right) / \Gamma=10$ (circles), the total measured noise reduces to twice the off-resonant SQL value because the thermal disturbance component $\left(S_{m}\right)$ drops faster than the SQL [Fig. 1(a)].

When measuring at a finite intermediate angle $\left(\phi=45^{\circ}\right)$, a distinct Fano-like line shape due to the cross-correlations appears [inset of Fig. 2(b)]. We see that the sensitivity is increased over a range of frequencies off resonance. Figures 2(b) and 3(a) show how a $\phi=45^{\circ}$ measurement (red) results in an imprecision below that at $\phi=90^{\circ}$ (blue) for frequencies above resonance and near the off-resonant SQL power. On the other hand, at low powers $(p \ll 1)$ there is no improvement because the $\mathrm{SN}$ contribution increases as one adds more AM noise that does not contain information about the resonator, and at high powers $(p \gg 1)$ the backaction contribution is dominant over the correlation and there is also no improvement.

In Fig. 2(b), we see the clear measurement improvement when detecting at $\left(\phi=45^{\circ}\right)$, and that its optimal power is at a higher power than for the standard measurement configuration $\left(\phi=90^{\circ}\right)$. For a given mechanical detuning $\left[\rho=2\left(\omega-\omega_{m}\right) / \Gamma\right]$, we find the optimal measurement quadrature to be $\cot \phi_{\text {opt }}=\epsilon p \rho\left|\tilde{\chi}_{m}(\rho)\right|^{2}$, which depends on both the quantum efficiency and measurement strength $(p)$. As derived in the Appendix, the achievable limit at the optimal power $\left(p_{\text {opt }}\right)$ depends on the quantum efficiency according to
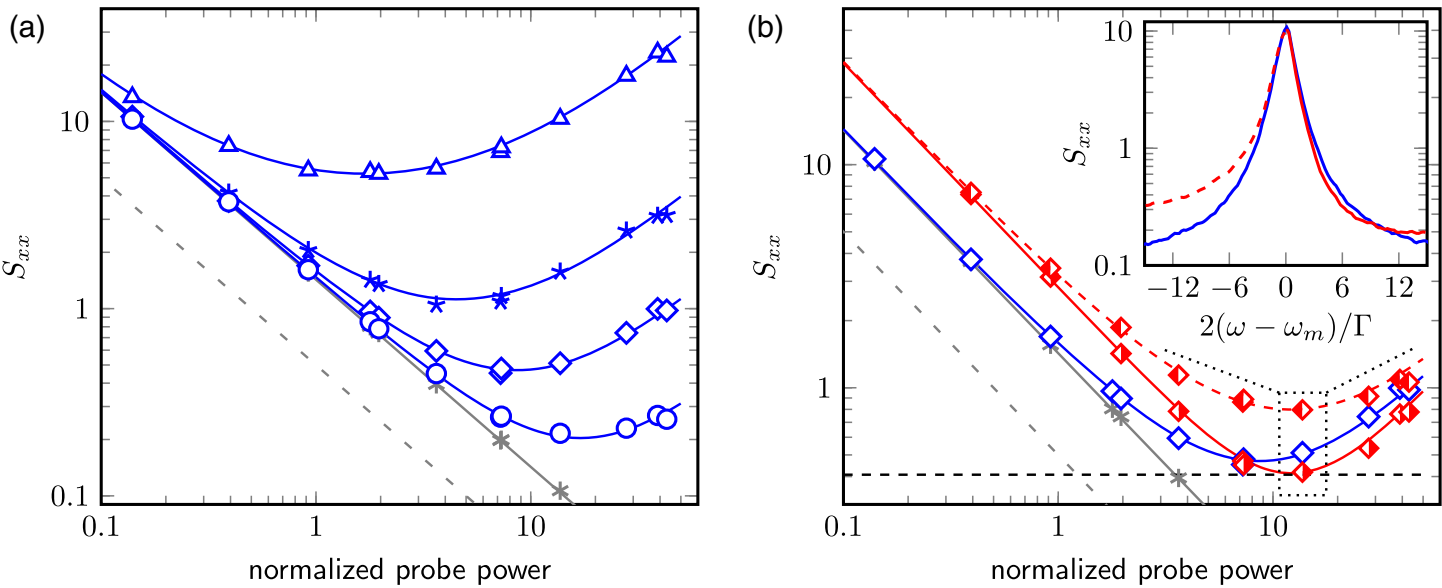

FIG. 2. Measured displacement PSD for different frequencies and measurement angles as a function of normalized probe power $p$. (a) Measurement at $\phi=90^{\circ}$ at different frequencies: $2\left(\omega-\omega_{m}\right) / \Gamma=0$ (triangles), 2.5 (stars), 5 (squares), 10 (circles). (b) Measurement at $\phi=90^{\circ}$ (blue) and at $\phi=45^{\circ}$ (red) compared to SN level at $\phi=90^{\circ}$ quadrature (gray). Measurement at $\phi=45^{\circ}$ at frequency $2\left(\omega-\omega_{m}\right) / \Gamma=-5$ (square filled on left, dashed red line) results in larger imprecision, and at frequency $2\left(\omega-\omega_{m}\right) / \Gamma=5($ square filled on right, solid red line) results in reduced imprecision. The dashed black line is the QL for $2\left(\omega-\omega_{m}\right) / \Gamma=5$ evaluated at quantum efficiency $\epsilon=0.35$, plus thermal contribution due to $n_{\text {th }}=1.29$. Red and blue lines are full power-dependent expectation for corresponding data points. Inset: Measured PSD as a function of frequency $2\left(\omega-\omega_{m}\right) / \Gamma$, at $\phi=90^{\circ}$ (blue) and $\phi=45^{\circ}$ (red) quadratures for power $p=14$, as indicated by the dotted box in main figure. For this choice of $\phi$, negative detunings (red dashed) yield worse sensitivity, while positive detunings (solid red) are improved. In both (a) and (b), the gray asterisks are SN measurement at $\phi=90^{\circ}$ for $\epsilon=0.35$, and the dashed gray line is for $\epsilon=1$. 

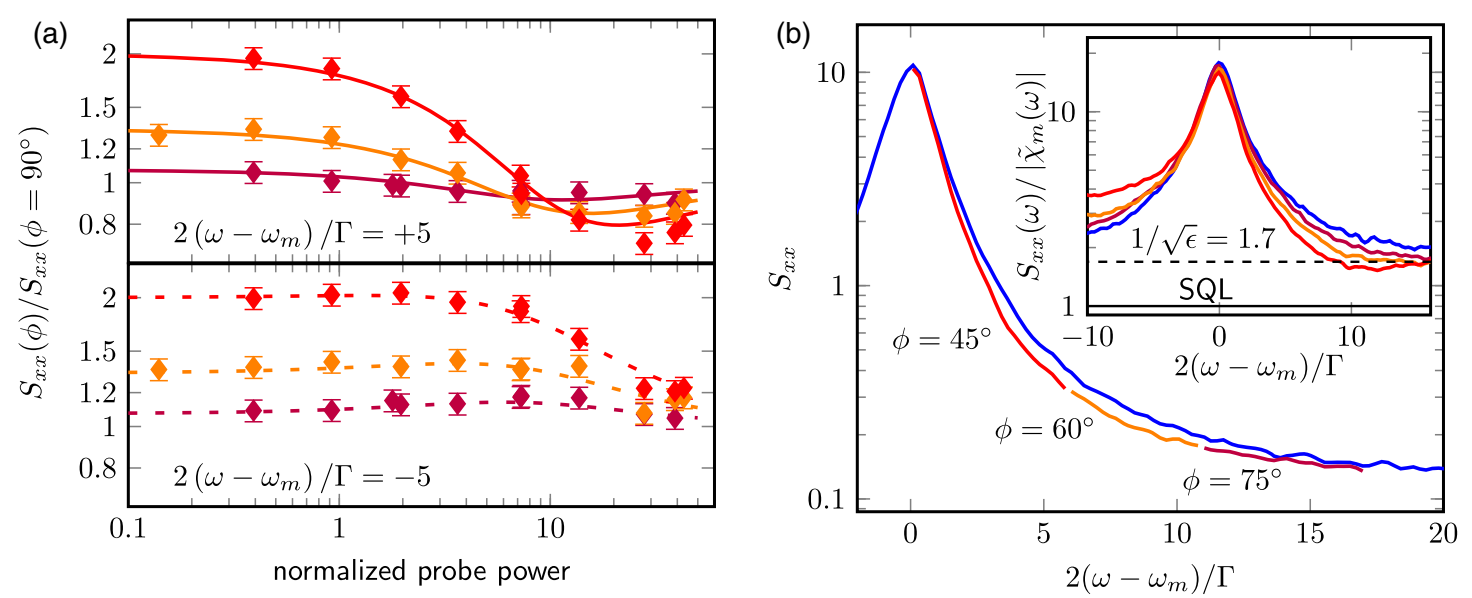

FIG. 3. Components of variational readout. (a) Ratio of PSD in quadratures $\phi=45^{\circ}$ (red), $\phi=60^{\circ}$ (orange), and $\phi=75^{\circ}$ (purple) to that in $\phi=90^{\circ}$ as a function of normalized probe power $p$. The top panel is at a detuning of $2\left(\omega-\omega_{m}\right) / \Gamma=5$, and the bottom panel at $2\left(\omega-\omega_{m}\right) / \Gamma=-5$. (b) Reconstruction of variational readout for $p=14$. The measured quadratures [same colors as (a)] are placed in the portion of the spectrum for which they provide the lowest imprecision. The result for $\phi=90^{\circ}$ (blue) is shown for comparison. Inset: Measured quadratures [same colors as (b)] at $p=28$ normalized to the corresponding SQL at each frequency.

$$
\begin{aligned}
S_{x x}\left(\rho, \phi_{\mathrm{opt}}, p_{\mathrm{opt}}\right)= & 2\left(n_{\mathrm{th}}+\frac{1}{2}\right)\left|\tilde{\chi}_{m}(\rho)\right|^{2} \\
& +\sqrt{\frac{1}{\epsilon}+\frac{1-\epsilon}{\epsilon} \rho^{2}}\left|\tilde{\chi}_{m}(\rho)\right|^{2},
\end{aligned}
$$

which is shown as the dashed black line in Fig. 2(b) for $\rho=5$. The above result at unit quantum efficiency reproduces the expected QL at all frequencies, as the amount of added noise is equal to the zero-point motion PSD contribution $\left[\left|\tilde{\chi}_{m}(\rho)\right|^{2}\right]$. In comparison, a typical measurement is at $\phi=90^{\circ}(\mathrm{PM})$ and will scale with quantum efficiency as $\left|\tilde{\chi}_{m}(\rho)\right| / \sqrt{\epsilon}$. Note that at unit efficiency this is the difference between QL and SQL.

In variational readout, the analysis quadrature would be changed as a function of frequency to realize an optimal measurement at all frequencies (reaching the $\mathrm{QL}$ at a single frequency). In our work, while we do not vary the quadrature in a single measurement, we are able to reconstruct variational readout spectra from four different measurements at different quadratures with the same power [Fig. 3(b)]. In the inset of Fig. 3(b), we show the data normalized to the corresponding off-resonant SQL at each frequency $\left[S_{\mathrm{SQL}}(\omega)\right]$ for an even larger probe power $(p=28)$, and we can quantitatively analyze our improvement compared to the SQL, under the constraint of finite quantum efficiency. At a frequency $2\left(\omega-\omega_{m}\right) / \Gamma=12$, we find $1.55 \pm 0.07$ times the SQL value for $\phi=45^{\circ}$. Taking into account our finite quantum efficiency, the minimum added noise we could hope to achieve for a $\phi=90^{\circ}$ measurement is $1 / \sqrt{\epsilon}=1.7$ times the SQL value and, hence, measurement at $\phi=45^{\circ}$ allows us to measure at $0.91 \pm 0.04$ times the finite quantum efficiency SQL. At this frequency the thermal and zero-point motion contribution of 0.3 (in units of off-resonance $S_{\mathrm{SQL}}$ ) is small, compared to the added noise due to the probe of 1.25 .

The technique we demonstrate shows the value and simplicity of utilizing imprecision-backaction correlations when carrying out strong measurement. In this technique the degree to which the SQL can be surpassed is greatly dependent on the quantum efficiency of the probe. In the future, pursuing higher quantum efficiency will be a natural goal of a variety of detectors and, hence, extend the utility of variational readout. High quantum efficiency combined with the ability to arbitrarily manipulate the local oscillator and corresponding correlations will offer useful opportunities to advance broadband displacement and force sensing beyond the standard quantum limit.

This work was supported by AFOSR PECASE, ONR DURIP, AFOSR-MURI, RAFAEL, the Cottrell Scholar program, and the National Science Foundation under Grant No. 1125844. We thank W. P. Bowen, T.P. Purdy, O. Wipfli, J.D. Teufel, and A. Higginbotham for useful conversation and expertise.

\section{APPENDIX: DERIVATIONS}

\section{Optomechanical interaction}

The optomechanical interaction in the interferometer is defined by the following Hamiltonian $[43,44]$ :

$$
\hat{H}_{0}=\frac{1}{4} \hbar \omega_{m}\left(\hat{x}^{2}+\hat{p}^{2}\right)+\hbar \omega_{c} \hat{a}^{\dagger} \hat{a}+\hbar g \hat{x} \hat{a}^{\dagger} \hat{a},
$$

where $\omega_{m}$ is the mechanical resonance frequency, $\hat{x}(\hat{p})$ is the mechanical position (momentum) fluctuation operator, 
$\omega_{c}$ is the optical cavity resonance frequency, $\hat{a}\left(\hat{a}^{\dagger}\right)$ is the optical intracavity annihilation (creation) operator, and $g$ is a single-photon optomechanical coupling constant. In this way of writing the Hamiltonian, the position and momentum operators are normalized to their zero-point fluctuations $x_{\mathrm{zp}}=\sqrt{\hbar / 2 m \omega_{m}}$ and $p_{\mathrm{zp}}=\sqrt{\frac{1}{2} m \hbar \omega_{m}}$. Here, $m$ is the mechanical resonator effective mass and $\hbar$ is the reduced Planck constant. In our analysis the Hamiltonian is linearized by assuming a large optical coherent state. Thus, we write the optical annihilation operator as $\hat{a}=\bar{a}+\hat{u}$, with $\bar{a}=\langle\hat{a}\rangle$, and neglecting the $\hat{u}^{\dagger} \hat{u}$ term.

\section{Heisenberg-Langevin equations for the light operators}

Here, we present the analysis of the HeisenbergLangevin equations for the probe light operator $u$, based on the Hamiltonian given above. Similar to previous analyses, we write the solution to the HeisenbergLangevin equations of motion of our optomechanical system $[33,43,45,46]$. In subsequent sections we convert the solutions to the units used in our final equations. In the Supplemental Material [35], we add to these equations a treatment of potential classical noise terms (and find them to be negligible for our experimental parameters).

The probe light quadratures $u_{\mathrm{AM}}$ and $u_{\mathrm{PM}}$ are given by

$$
\begin{aligned}
\left(\begin{array}{c}
\hat{u}_{\mathrm{AM}}(\omega) \\
\hat{u}_{\mathrm{PM}}(\omega)
\end{array}\right) & =\left(\begin{array}{c}
\hat{\mu}_{\mathrm{AM}}(\omega) \\
\hat{\mu}_{\mathrm{PM}}(\omega)
\end{array}\right) \\
& +\sqrt{\epsilon \kappa} g \bar{a}\left(\begin{array}{cc}
\pi_{-}(\omega) & 0 \\
0 & \pi_{+}(\omega)
\end{array}\right)\left(\begin{array}{c}
\hat{x}(\omega) \\
\hat{x}(\omega)
\end{array}\right)
\end{aligned}
$$

here, $\hat{\mu}_{\mathrm{AM}}(\omega)$ and $\hat{\mu}_{\mathrm{PM}}(\omega)$ are the light shot-noise Langevin operators for the amplitude (AM) $\phi=0^{\circ}$ ) and phase (PM) $\left(\phi=90^{\circ}\right)$ quadratures, $\bar{a}$ is the intracavity coherent state amplitude, $\epsilon$ is the quantum efficiency, $\hat{x}(\omega)$ is the resonator state, and $\pi_{ \pm}(\omega)$ are the constructive and destructive cavity susceptibility interference functions defined as $\pi_{+}(\omega)=\chi_{c}^{*}(-\omega)+\chi_{c}(\omega)$ and $\pi_{-}(\omega)=i\left[\chi_{c}^{*}(-\omega)-\chi_{c}(\omega)\right]$. $\chi_{c}(\omega)$ is the cavity susceptibility given by $\chi_{c}(\omega)=[\kappa / 2-i(\omega+\Delta)]^{-1}$, with $\Delta$ the probe detuning relative to the cavity resonance and $\kappa$ the optical cavity linewidth.

The measured light operator at a given phase $\phi$ is given by $\hat{u}_{\phi}=\hat{u}_{\mathrm{AM}} \cos \phi-\hat{u}_{\mathrm{PM}} \sin \phi$. We use this to calculate the symmetrized light PSD, $S_{\phi}(\omega, \phi)=\left\langle\hat{u}_{\phi}(-\omega) \hat{u}_{\phi}(\omega)\right\rangle$ $[4,43,47]$. Then the dimensionless $S_{\phi}$ is decomposed as follows:

$$
S_{\phi}(\omega, \phi)=1+f_{x x}(\omega, \phi)\langle\hat{x} \hat{x}\rangle(\omega)+S_{\mu \hat{x}}(\omega, \phi),
$$

where 1 is the probe shot noise, $f_{x x}(\omega, \phi)$ is the transfer function from displacement to light, $\langle\hat{x} \hat{x}\rangle(\omega)$ is the symmetrized resonator displacement distribution PSD, and $S_{\mu \hat{x}}(\omega, \phi)$ is the cross-correlation PSD between the light shot noise and the resonator state as inferred by the probe.

To explicitly write the above functions, we define the following cavity and light parameters: $n_{\text {th }}$ is the thermal phonon occupation, $\Gamma$ the effective mechanical linewidth, $\omega_{m}$ the resonator frequency, and the mechanical susceptibility is given by $\chi_{m}(\omega)=\left[\Gamma / 2-i\left(\omega-\omega_{m}\right)\right]^{-1}$. We find

$$
\begin{gathered}
f_{x x}(\omega, \phi)=\epsilon \kappa(g \bar{a})^{2}\left\{\left|\chi_{c}(-\omega)\right|^{2}+\left|\chi_{c}(\omega)\right|^{2}\right\} \\
-2 \epsilon \kappa(g \bar{a})^{2} \operatorname{Re}\left[\chi_{c}(-\omega) \chi_{c}(\omega) e^{-2 i \phi}\right], \quad(\mathrm{A} 4) \\
S_{\mu \hat{x}}(\omega, \phi)=\epsilon \kappa(g \bar{a})^{2}\left[\left|\chi_{c}(-\omega)\right|^{2}-\left|\chi_{c}(\omega)\right|^{2}\right] \operatorname{Im}\left[i \chi_{m}(\omega)\right] \\
-2 \epsilon \kappa(g \bar{a})^{2} \operatorname{Im}\left[\chi_{c}(-\omega) \chi_{c}(\omega) e^{-2 i \phi}\right] \operatorname{Re}\left[i \chi_{m}(\omega)\right],
\end{gathered}
$$

$$
\begin{aligned}
\langle\hat{x} \hat{x}\rangle(\omega)= & \Gamma\left(n_{\mathrm{th}}+1 / 2\right)\left|\chi_{m}(\omega)\right|^{2} \\
& +(g \bar{a})^{2}\left|\chi_{m}(\omega)\right|^{2} \frac{\kappa}{2}\left[\left|\chi_{c}(-\omega)\right|^{2}+\left|\chi_{c}(\omega)\right|^{2}\right] \\
& +\left(\frac{F}{4 p_{\text {zp }}}\right)^{2}\left|\chi_{m}(\omega)\right|^{2} \delta\left(\omega-\omega_{f}\right) .
\end{aligned}
$$

In Eq. (A6), we include the response to external force $(F)$, applied on the resonator at a frequency $\omega_{f}$. Note that the $\Gamma$ and $\omega_{m}$ are effective mechanical parameters due to wellknown optical damping and spring effects dominantly from a red-detuned damping tone but with a very small contribution from the small detuning of the probe [44].

\section{Derivation of the standard quantum limit value}

Here, we derive the SQL PSD $\left(S_{\mathrm{SQL}}\right)$ using the result above by minimizing the combined shot-noise and quantum backaction terms [3].

$$
S_{\mathrm{SQL}}(\omega)=x_{\mathrm{zp}}^{2} \min _{\bar{a}, \phi, \Delta}\left\{\frac{1}{f_{x x}(\omega, \phi)}+(g \bar{a})^{2}\left|\chi_{m}(\omega)\right|^{2} \frac{\kappa}{2}\left[\left|\chi_{c}(-\omega)\right|^{2}+\left|\chi_{c}(\omega)\right|^{2}\right]\right\},
$$

with $f_{x x}(\omega, \phi)$ the transfer function from displacement to light (with units of hertz) of Eq. (A4). The $S_{\mathrm{SQL}}$ is composed only of the added noise by the measurement probe (shot noise and quantum backaction), and ignores the mechanical state (thermal and zero-point motion). This is motivated from the point of view that the mechanical 
resonator state is the signal to measure. We note that in Ref. [11] the zero-point motion is included in the total noise.

The minimum value of the SQL PSD is found for the detection angle of $\phi=90^{\circ}$ and for probing on cavity resonance $(\Delta=0)$. Additionally, we evaluate $f_{x x}$ for unity detection efficiency $(\epsilon=1)$. With this we find the number of photons required for SQL detection to be

$$
\begin{aligned}
\bar{a}_{\mathrm{SQL}}^{2}(\omega) & =\frac{1}{2 \kappa g^{2}\left|\chi_{c}(\omega)\right|^{2}\left|\chi_{m}(\omega)\right|} \\
& =\frac{\Gamma}{4 \kappa g^{2}\left|\chi_{c}(\omega)\right|^{2}} \sqrt{1+\rho^{2}},
\end{aligned}
$$

with $\rho=2\left[\left(\omega-\omega_{m}\right) / \Gamma\right]$ a dimensionless mechanical detuning. We combine this result with Eq. (A7) to find

$$
S_{\mathrm{SQL}}(\omega)=x_{\mathrm{zp}}^{2}\left|\chi_{m}(\omega)\right|=x_{\mathrm{zp}}^{2} \frac{2}{\Gamma} \frac{1}{\sqrt{1+\rho^{2}}} .
$$

When evaluated on resonance the SQL value is $S_{\mathrm{SQL}}\left(\omega_{m}\right)=\left[\hbar /\left(m \omega_{m} \Gamma\right)\right]$. We utilize a probe power $(p)$ normalized to the on-resonant SQL power; i.e., $p=\left\{\bar{a}^{2} /\left[\bar{a}_{\mathrm{SQL}}^{2}\left(\omega_{m}\right)\right]\right\}$. Note that $p$ is related to the commonly used optomechanical cooperativity $(C)$ by $C=$ $p / 4[11,44]$.

\section{Converting measurement noise results to dimensionless displacement PSD}

In this section, we write the result of the HeisenbergLangevin equations for the light operators inferred as displacement of the resonator and use it to evaluate the displacement PSD. While the light equations and associated PSD have been derived many times in the literature $[33,43,45,46]$, our approach is to use quantum noise notation, as described in, for example, Ref. [4], which allows us to see more transparently how the QL can be reached for a particular optical probe configuration.

The probe light operator converted to inferred displacement $\hat{X}$ is given by multiplying the probe light operator $\hat{u}$ by $x_{\mathrm{zp}} / \sqrt{f_{x x}\left(\phi=90^{\circ}\right)} \quad\left(=x_{\mathrm{zp}} / \sqrt{\epsilon \Gamma p}\right)$, which gives dimensions of $\mathrm{m} / \sqrt{\mathrm{Hz}}$. We normalize the displacement by the on-resonance SQL amplitude $\left[\sqrt{S_{\mathrm{SQL}}\left(\omega_{m}\right)}\right]$. We also use a dimensionless mechanical susceptibility defined by $\tilde{\chi}_{m}(\rho)=\chi_{m}(\omega) /\left|\chi_{m}\left(\omega_{m}\right)\right|=(1-i \rho)^{-1}$ and a dimensionless cavity susceptibility $\tilde{\chi}_{c}(\omega)=\chi_{c}(\omega) /\left|\chi_{c}\left(\omega_{m}\right)\right|$. With this, we write the AM and PM quadrature operators for the inferred displacement as

$$
\begin{gathered}
\hat{X}_{\mathrm{AM}}(\omega)=\frac{1}{\sqrt{2 \epsilon p} \tilde{\chi}_{c}(\omega)} \hat{\mu}_{\mathrm{AM}}(\omega), \\
\hat{X}_{\mathrm{PM}}(\omega)=\frac{1}{\sqrt{2 \epsilon p} \tilde{\chi}_{c}(\omega)} \hat{\mu}_{\mathrm{PM}}(\omega)+\sqrt{\frac{\Gamma}{2}} \hat{x}(\omega),
\end{gathered}
$$

$\sqrt{\frac{\Gamma}{2}} \hat{x}(\omega)=\hat{x}_{m}(\omega)-2 \omega_{m} \chi_{m}^{*}(-\omega) \tilde{\chi}_{m}(\omega) \hat{F}_{\mathrm{ba}}(\omega)$,

with $\hat{x}_{m}$ the resonator state (which includes zero-point motion, thermal and external force) and $\hat{F}_{\text {ba }}$ the dimensionless backaction force applied onto the resonator. The backaction operator is proportional to $\sqrt{p}$ and is a function of the shot-noise AM quadrature operator $[33,43]$.

Because the resonator $(\hat{x})$ information is only in the PM quadrature, the displacement phase dependence is given by

$$
\begin{aligned}
\hat{X}_{\phi}(\omega) & =-\cot \phi \hat{X}_{\mathrm{AM}}(\omega)+\hat{X}_{\mathrm{PM}}(\omega) \\
& =\hat{x}_{m}(\omega)+\hat{I}_{\phi}-2 \omega_{m} \tilde{\chi}_{m}^{*}(-\omega) \tilde{\chi}_{m}(\omega) \hat{F}_{\mathrm{ba}}(\omega) \\
& \cong \hat{x}_{m}(\omega)+\hat{I}_{\phi}-i \tilde{\chi}_{m}(\omega) \hat{F}_{\mathrm{ba}}(\omega) .
\end{aligned}
$$

Here, we define the shot-noise displacement imprecision operator to be $\hat{I}_{\phi}=\left\{1 /\left[\sqrt{2 \epsilon p} \tilde{\chi}_{c}(\omega)\right\}\left[-\cot \phi \hat{\mu}_{\mathrm{AM}}(\omega)+\right.\right.$ $\left.\hat{\mu}_{\mathrm{PM}}(\omega)\right]$, from which it follows that there is a weak frequency dependence proportional to $\left|1 / \tilde{\chi}_{c}(\omega)\right|$. In the third line we take the limit of a high- $Q$ resonator $\left(\Gamma \ll \omega_{m}\right)$. Note that in $\mathrm{AM} \cot \phi=\cot 0=\infty$ corresponds to the case in which all the information about the resonator is in the $\phi=90^{\circ}$ (PM) quadrature and, thus, the displacement measurement diverges. It follows that the displacement PSD is given by

$$
\begin{aligned}
S_{x x}(\omega)= & \left\langle\hat{X}_{\phi}(-\omega) \hat{X}_{\phi}(\omega)\right\rangle \\
\cong & S_{m}(\omega)+S_{I I}(\omega)+\left|\tilde{\chi}_{m}(\rho)\right|^{2} S_{F F}(\omega) \\
& +2 \operatorname{Im}\left[\tilde{\chi}_{m}(\rho) S_{I F}\right],
\end{aligned}
$$

with functions given by

$$
\begin{gathered}
S_{m}(\omega)=2\left(n_{\mathrm{th}}+\frac{1}{2}\right)\left|\tilde{\chi}_{m}(\rho)\right|^{2}, \\
S_{I I}(\omega)=\frac{1+\cot ^{2} \phi}{2 \epsilon p\left|\tilde{\chi}_{c}(\omega)\right|^{2}} \\
S_{F F}(\omega)=\frac{1}{2} p\left|\tilde{\chi}_{c}(\omega)\right|^{2}, \\
S_{I F}(\omega)=-\frac{1}{2} \cot \phi .
\end{gathered}
$$

Here, $S_{m}$ is the displacement PSD of the resonator including thermal and zero-point motion, in which we set the external force to zero, $S_{I I}$ is the displacement imprecision PSD due to the probe $\mathrm{SN}$ and is inversely proportional to the power, $S_{F F}$ is the displacement change due to the backaction force, $S_{I F}$ is the cross-correlation term measured in homodyne detection and is real, but in general it can be complex [4]. For example, in synodyne detection the equivalent $S_{I F}$ is complex and $S_{I F}=-S_{F I}^{*}[34]$. 
When examining the contribution of the backaction in Eq. (A13), there is a $90^{\circ}$ phase delay with respect to the shot-noise term $\left(\hat{I}_{\phi}\right)$. This means there is a time lag between the backaction force applied on the resonator and the measurement time. For this reason, there must be a frequency dependence in the cross-correlation term. Only the imaginary part of the mechanical susceptibility $\operatorname{Im} \tilde{\chi}_{m}(\rho)=\rho\left|\tilde{\chi}_{m}(\rho)\right|^{2}$ contributes to the PSD.

It is also useful to explicitly write out the PSD with the assumption $\left|\tilde{\chi}_{c}(\omega)\right|^{2}=1$, which is a very good approximation for our experimental parameters:

$$
\begin{aligned}
S_{x x}(\omega, p, \phi)= & 2\left(n_{\mathrm{th}}+\frac{1}{2}\right)\left|\tilde{\chi}_{m}(\omega)\right|^{2}+\frac{1+\cot ^{2} \phi}{2 \epsilon p} \\
& +\frac{1}{2} p\left|\tilde{\chi}_{m}(\omega)\right|^{2}-\cot \phi \rho\left|\tilde{\chi}_{m}(\omega)\right|^{2} .
\end{aligned}
$$

\section{Comparison to uncertainty relations and parameters for reaching the $\mathrm{QL}$}

Through Eqs. (A14)-(A19) we can compare the PSD to the Heisenberg uncertainty relation [28] for the state of probe light and to the SQL. The Heisenberg uncertainty relation is given by

$$
\begin{aligned}
\Delta \hat{I}_{\phi}^{2} \Delta \hat{F}_{\mathrm{ba}}^{2} & \geq \frac{1}{4}\left|\left\langle\left[\hat{I}_{\phi}, \hat{F}_{\mathrm{ba}}\right]\right\rangle\right|^{2}+\frac{1}{4}\left|\left\langle\left\{\hat{I}_{\phi}, \hat{F}_{\mathrm{ba}}\right\}\right\rangle\right|^{2}, \\
S_{I I} S_{F F} & \geq \frac{1}{4}+S_{I F}^{2} .
\end{aligned}
$$

For $\epsilon=1$ and $\phi=90^{\circ}, S_{I I} S_{F F}=\frac{1}{4}$, which is the case for a SQL measurement configuration, and is power independent. But when measuring at some intermediate angle $\left(0^{\circ}<\phi<90^{\circ}\right)$, the measurement imprecision can be below the SQL value of $1 / 4$.

We can also determine the correct choice of $p$ and $\phi$ to reach the QL, when the additional contribution of the crosscorrelation term $\left(S_{I F}\right)$ is taken into account. When we optimize the PSD [Eq. (A19)] to find the optimal measurement phase, we find $\cot \phi_{\text {opt }}=\epsilon p \rho\left|\tilde{\chi}_{m}(\rho)\right|^{2}$. Placing this back into Eq. (A19), we find the succinct consequence of variational readout,

$$
\begin{aligned}
S_{x x}\left(\rho, \phi_{\mathrm{opt}}, p\right)= & 2\left(n_{\mathrm{th}}+\frac{1}{2}\right)\left|\tilde{\chi}_{m}(\rho)\right|^{2} \\
& +\frac{1}{2 \epsilon p}+\frac{1}{2} p\left[1+(1-\epsilon) \rho^{2}\right]\left|\tilde{\chi}_{m}(\rho)\right|^{4},
\end{aligned}
$$

for a given quantum efficiency.

At the optimal power $p_{\text {opt }}$ and optimal angle, one finds precisely the QL:

$$
\begin{aligned}
& S_{x x}\left(\rho, \phi_{\text {opt }}, p_{\text {opt }}\right)= 2\left(n_{\text {th }}+\frac{1}{2}\right)\left|\tilde{\chi}_{m}(\rho)\right|^{2} \\
&+\sqrt{\frac{1}{\epsilon}+\frac{1-\epsilon}{\epsilon} \rho^{2}}\left|\tilde{\chi}_{m}(\rho)\right|^{2}, \\
& p_{\text {opt }}=\frac{1}{\sqrt{\epsilon\left[1+(1-\epsilon) \rho^{2}\right]}\left|\tilde{\chi}_{m}(\rho)\right|^{2}} .
\end{aligned}
$$

We recall that the QL for an ideal detector reaches one zeropoint motion contribution for each measured mechanical quadrature [black lines in Fig. 1(a)].

\section{Comparison to ponderomotive squeezing}

The above analysis gives us the necessary tools to directly compare the consequence of variational readout on displacement sensitivity to the creation of ponderomotive squeezing. Namely, we can compare the $S_{\phi}$ derived in Appendix A 2 to $S_{x x}$ to find that the light PSD has a different $\phi$ dependence than the displacement PSD [31-33]. We can write this as

$$
S_{\phi}(\rho, \phi, p)=2 \epsilon p \sin ^{2} \phi S_{x x}(\rho, \phi, p) .
$$

The different phase dependencies are illustrated in the main text in Fig. 1(d). For light squeezing, the information about the resonator does not matter. Conversely, for displacement measurement, while the larger cross-correlation term reduces the backaction contribution by rotating towards the AM quadrature, it also dilutes mechanical information found in the PM quadrature. For this reason, the optimal ponderomotive squeezing angle is closer to the AM quadrature than for the displacement measurement.

[1] H. A. Haus and J. A. Mullen, Quantum Noise in Linear Amplifiers, Phys. Rev. 128, 2407 (1962).

[2] C. M. Caves, Quantum Limits on Noise in Linear Amplifiers, Phys. Rev. D 26, 1817 (1982).

[3] V. B. Braginsky, M. L. Gorodetsky, F. Ya. Khalili, A. B. Matsko, K.S. Thorne, and S.P. Vyatchanin, Noise in Gravitational-Wave Detectors and Other Classical-Force Measurements Is Not Influenced by Test-Mass Quantization, Phys. Rev. D 67, 082001 (2003).

[4] A. A. Clerk, M. H. Devoret, S. M. Girvin, F. Marquardt, and R.J. Schoelkopf, Introduction to Quantum Noise, Measurement, and Amplification, Rev. Mod. Phys. 82, 1155 (2010).

[5] M. T. Jaekel and S. Reynaud, Quantum Limits in Interferometric Measurements, Europhys. Lett. 13, 301 (1990).

[6] V. B. Braginskii and Yu. I. Vorontsov, Classical and Quantum Restrictions on the Detection of Weak Disturbances of a Macroscopic Oscillator, Sov. Phys. JETP 26, 831 (1968).

[7] C. M. Caves, Quantum-Mechanical Radiation-Pressure Fluctuations in an Interferometer, Phys. Rev. Lett. 45, 75 (1980). 
[8] M. D. LaHaye, O. Buu, B. Camarota, and K. C. Schwab, Approaching the Quantum Limit of a Nanomechanical Resonator, Science 304, 74 (2004).

[9] T. P. Purdy, R. W. Peterson, and C. A. Regal, Observation of Radiation Pressure Shot Noise on a Macroscopic Object, Science 339, 801 (2013).

[10] A. H. Safavi-Naeini, J. Chan, J. T. Hill, Thiago P. Mayer Alegre, A. Krause, and O. Painter, Observation of Quantum Motion of a Nanomechanical Resonator, Phys. Rev. Lett. 108, 033602 (2012).

[11] S. Schreppler, N. Spethmann, N. Brahms, T. Botter, M. Barrios, and D. M. Stamper-Kurn, Optically Measuring Force Near the Standard Quantum Limit, Science 344, 1486 (2014).

[12] J. D. Teufel, F. Lecocq, and R. W. Simmonds, Overwhelming Thermomechanical Motion with Microwave Radiation Pressure Shot Noise, Phys. Rev. Lett. 116, 013602 (2016).

[13] H. Miao, H. Yang, R. X. Adhikari, and Y. Chen, Quantum Limits of Interferometer Topologies for Gravitational Radiation Detection, Classical Quantum Gravity 31, 165010 (2014).

[14] S. P. Vyatchanin and E. A. Zubova, Quantum Variation Measurement of a Force, Phys. Lett. A 201, 269 (1995).

[15] H. J. Kimble, Y. Levin, A. B. Matsko, K. S. Thorne, and S. P. Vyatchanin, Conversion of Conventional GravitationalWave Interferometers into Quantum Nondemolition Interferometers by Modifying Their Input and/or Output Optics, Phys. Rev. D 65, 022002 (2001).

[16] V. B. Braginsky, Y. I. Vorontsov, and K. S. Thorne, Quantum Nondemolition Measurements, Science 209, 547 (1980).

[17] K. Hammerer, M. Aspelmeyer, E. S. Polzik, and P. Zoller, Establishing Einstein-Poldosky-Rosen Channels between Nanomechanics and Atomic Ensembles, Phys. Rev. Lett. 102, 020501 (2009).

[18] M. Tsang and C. M. Caves, Evading Quantum Mechanics: Engineering a Classical Subsystem within a Quantum Environment, Phys. Rev. X 2, 031016 (2012).

[19] C. F. Ockeloen-Korppi, E. Damskagg, J.-M. Pirkkalainen, A. A. Clerk, M. J. Woolley, and M. A. Sillanpaa, Quantum Backaction Evading Measurement of Collective Mechanical Modes, Phys. Rev. Lett. 117, 140401 (2016).

[20] C. B. Moller, R. A. Thomas, G. Vasilakis, E. Zeuthen, Y. Tsaturyan, K. Jensen, A. Schliesser, K. Hammerer, and E. S. Polzik, Back Action Evading Quantum Measurement of Motion in a Negative Mass Reference Frame, arXiv:1608.03613.

[21] J. Suh, A. J. Weinstein, C. U. Lei, E. E. Wollman, S. K. Steinke, P. Meystre, A. A. Clerk, and K. C. Schwab, Mechanically Detecting and Avoiding the Quantum Fluctuations of a Microwave Field, Science 344, 1262 (2014).

[22] E. E. Wollman, C. U. Lei, A. J. Weinstein, J. Suh, A. Kronwald, F. Marquardt, A. A. Clerk, and K. C. Schwab, Quantum Squeezing of Motion in a Mechanical Resonator, Science 349, 952 (2015).

[23] F. Lecocq, J. B. Clark, R. W. Simmonds, J. Aumentado, and J. D. Teufel, Quantum Nondemolition Measurement of a Nonclassical State of a Massive Object, Phys. Rev. X 5, 041037 (2015).

[24] J.-M. Pirkkalainen, E. Damskägg, M. Brandt, F. Massel, and M. A. Sillanpää, Squeezing of Quantum Noise of Motion in a Micromechanical Resonator, Phys. Rev. Lett. 115, 243601 (2015).

[25] H. Rehbein, J. Harms, R. Schnabel, and K. Danzmann, Optical Transfer Functions of Kerr Nonlinear Cavities and Interferometers, Phys. Rev. Lett. 95, 193001 (2005).

[26] C. Laflamme and A. A. Clerk, Quantum-Limited Amplification with a Nonlinear Cavity Detector, Phys. Rev. A 83, 033803 (2011).

[27] V. B. Braginsky and F. Y. Khalili, Quantum Measurement (Cambridge University Press, Cambridge, England, 1992).

[28] H. P. Robertson, An Indeterminacy Relation for Several Observables and Its Classical Interpretation, Phys. Rev. 46, 794 (1934).

[29] W. Heisenberg, The Actual Content of Quantum Theoretical Kinematics and Mechanics, Z. Phys. 43, 172 (1927).

[30] V. Sudhir, D. J. Wilson, R. Schilling, H. Schütz, S. A. Fedorov, A. H. Ghadimi, A. Nunnenkamp, and T. J. Kippenberg, Appearance and Disappearance of Quantum Correlations in Measurement-Based Feedback Control of a Mechanical Oscillator, Phys. Rev. X 7, 011001 (2017).

[31] D. W. C. Brooks, T. Botter, S. Schreppler, T. P. Purdy, N. Brahms, and D. M. Stamper-Kurn, Non-Classical Light Generated by Quantum-Noise-Driven Cavity Optomechanics, Nature (London) 488, 476 (2012).

[32] A. H. Safavi-Naeini, S. Groblacher, J. T. Hill, J. Chan, M. Aspelmeyer, and O. Painter, Squeezed Light from a Silicon Micromechanical Resonator, Nature (London) 500, 185 (2013).

[33] T. P. Purdy, P.-L. Yu, R. W. Peterson, N. S. Kampel, and C. A. Regal, Strong Optomechanical Squeezing of Light, Phys. Rev. X 3, 031012 (2013).

[34] L. F. Buchmann, S. Schreppler, J. Kohler, N. Spethmann, and D. M. Stamper-Kurn, Complex Squeezing and Force Measurement Beyond the Standard Quantum Limit, Phys. Rev. Lett. 117, 030801 (2016).

[35] See Supplemental Material at http://link.aps.org/ supplemental/10.1103/PhysRevX.7.021008 for details of the experimental protocol, optomechanical coupling coefficient calibrations, assessment of classical noise, and comparison to synodyne detection.

[36] J. B. Clark, F. Lecocq, R. W. Simmonds, J. Aumentado, and J. D. Teufel, Observation of Strong Radiation Pressure Forces from Squeezed Light on a Mechanical Oscillator, Nat. Phys. 12, 683 (2016).

[37] R. Schnabel, N. Mavalvala, D. E. McClelland, and P. K. Lam, Quantum Metrology for Gravitational Wave Astronomy, Nat. Commun. 1, 121 (2010).

[38] LIGO Scientific Collaboration, A Gravitational Wave Observatory Operating Beyond the Quantum Shot-Noise Limit, Nat. Phys. 7, 962 (2011).

[39] J. D. Thompson, B. M. Zwickl, A. M. Jayich, Florian Marquardt, S. M. Girvin, and J. G. E. Harris, Strong Dispersive Coupling of a High-Finesse Cavity to a Micromechanical Membrane, Nature (London) 452, 72 (2008).

[40] R. W. Peterson, T. P. Purdy, N. S. Kampel, R. W. Andrews, P.-L. Yu, K. W. Lehnert, and C. A. Regal, Laser Cooling of a Micromechanical Membrane to the Quantum Backaction Limit, Phys. Rev. Lett. 116, 063601 (2016).

[41] P.-L. Yu, K. Cicak, N. S. Kampel, Y. Tsaturyan, T. P. Purdy, R. W. Simmonds, and C. A. Regal, A Phononic Bandgap 
Shield for High-q Membrane Microresonators, Appl. Phys. Lett. 104, 023510 (2014).

[42] Y. Tsaturyan, A. Barg, A. Simonsen, L. G. Villanueva, S. Schmid, A. Schliesser, and E. S. Polzik, Demonstration of Suppressed Phonon Tunneling Losses in Phononic Bandgap Shielded Membrane Resonators for High-q Optomechanics, Opt. Express 22, 6810 (2014).

[43] K. Børkje, A. Nunnenkamp, B. M. Zwickl, C. Yang, J. G. E. Harris, and S. M. Girvin, Observability of RadiationPressure Shot Noise in Optomechanical Systems, Phys. Rev. A 82, 013818 (2010).

[44] M. Aspelmeyer, T. J. Kippenberg, and F. Marquardt, Cavity Optomechanics, Rev. Mod. Phys. 86, 1391 (2014).
[45] A. J. Weinstein, C. U. Lei, E. E. Wollman, J. Suh, A. Metelmann, A. A. Clerk, and K. C. Schwab, Observation and Interpretation of Motional Sideband Asymmetry in a Quantum Electromechanical Device, Phys. Rev. X 4, 041003 (2014).

[46] T. Botter, D. W. C. Brooks, N. Brahms, S. Schreppler, and D. M. Stamper-Kurn, Linear Amplifier Model for Optomechanical Systems, Phys. Rev. A 85, 013812 (2012).

[47] A. A. Clerk, Quantum Machines: Measurement and Control of Engineered Quantum Systems, Proceedings of the Les Houches Summer School (Oxford University Press, Oxford, 2014). 